\title{
El eportfolio una herramienta para la evaluación del desarrollo profesional docente
}

\author{
Alicia Martínez De la Muela \\ alicia.martinez@unir.net \\ Universidad Internacional de La Rioja (UNIR), Spain \\ Blanca Arteaga Martínez \\ blanca.arteaga@uah.es \\ Universidad de Alcalá, Spain \\ Mercedes García García \\ mergarci@ucm.es \\ Universidad Complutense de Madrid, Spain
}

\begin{abstract}
Resumen
El interés para determinar un proceso de evaluación del desarrollo profesional de los docentes ha cobrado especial interés en las últimas décadas por considerarse un factor de eficacia de los sistemas educativos. El hecho de que se constituya como un proceso continuo nos lleva a un planteamiento evaluador que toma como indicador la autopercepción de un conjunto de competencias genéricas ligadas a la mejora (reflexiva, autonomía, razonamiento crítico y trabajo equipo).

Este estudio utiliza la herramienta digital eportfolio con una muestra de docentes $(n=306)$ de educación obligatoria en dos cursos de formación. Los análisis cuantitativos y cualitativos permiten concluir que el eportfolio es útil en el desarrollo profesional porque facilita la reflexión crítica; sin embargo, no se obtienen mejoras significativas en la percepción de las competencias de autonomía y trabajo en equipo. Estos resultados nos llevan a plantear una propuesta de intervención en la elaboración y uso de la herramienta eportfolio en la formación docente.
\end{abstract}

\section{Palabras clave}

Evaluación; eportfolio; profesorado; desarrollo profesional; competencias 


\title{
The eportfolio a tool for the evaluation of the professional development teacher
}

\author{
Alicia Martínez De la Muela \\ alicia.martinez@unir.net \\ Universidad Internacional de La Rioja (UNIR), Spain \\ Blanca Arteaga Martínez \\ blanca.arteaga@uah.es \\ Universidad de Alcalá, Spain

\section{Mercedes García García} \\ mergarci@ucm.es \\ Universidad Complutense de Madrid, Spain
}

\begin{abstract}
The interest to determine a process of evaluation of the professional development of the teachers has gained special interest in the last decades because it is considered a factor of effectiveness of the educational systems. The fact that it is constituted as a continuous and evolutionary process, leads us to an evaluative approach that takes as an indicator the self-perception of a set of generic competences (reflexive, autonomy, critical reasoning and team work).

This study uses the eportfolio digital tool with a sample of teachers $(n=306)$ of compulsory education in two training courses. The quantitative and qualitative analysis allow us to conclude that the eportfolio is useful in professional development because it facilitates critical reflection; However, there are no significant improvements in autonomy and teamwork skills. These results lead us to propose a proposal for intervention in the development and use of the eportfolio tool in teacher training.
\end{abstract}

\section{Keywords}

Evaluation; eportfolio; teachers; professional development; competences 


\section{Introducción}

El desarrollo profesional docente se define como un proceso de aprendizaje que abarca toda la vida del profesorado, entre la que se incluye la formación de docentes en ejercicio y la autoformación (Tejada Fernández, 2013). El desarrollo profesional se caracteriza por ser continuo, conduciendo al docente a mejorar su práctica educativa (Day, 1999; González Maura, 2006) en contextos de aprendizaje adaptados a la realidad de los sistemas educativos actuales. La puesta en marcha de metodologías adaptadas al alumnado y la inclusión de recursos digitales lleva a plantear una formación apropiada para el cambio. La innovación educativa requiere de docentes comprometidos por la educación, considerándola como "una actualización constante en su formación para adaptarse a los cambios en la sociedad" (Iglesias, Lozano y Roldán, 2018, p. 29) y lograr las condiciones necesarias para el desarrollo de las potencialidades del alumnado a través de la autonomía para selección, organización y transmisión de contenidos (Carbonell, 2012). La formación docente centrada en el desarrollo profesional se dirige hacia el logro de "conocimientos, destrezas y actitudes para desarrollar profesionales reflexivos o investigadores" (Imbernón, 1999, p. 65) que les hagan adaptarse al contexto de aprendizaje actual y, por tanto, en el desarrollo de unas competencias básicas relacionadas con el compromiso personal para la mejora educativa.

Uno de los elementos clave para la consolidación de la innovación educativa y que influye en la práctica docente, es transformar el papel del docente como observador y evaluador de su propio proceso de aprendizaje. "La innovación de la evaluación es una consecuencia lógica del planteamiento de la formación como desarrollo de competencias y, por tanto, es un condicionante imprescindible para la innovación de la formación" (Villardón, 2006, p. 60). En este sentido, se habla de evaluación referida a aspectos reflexivos y organizativos (Cabero, Llorente y MoralesLozano, 2018) que irán guiando su aprendizaje si se fomenta la autorregulación.

El docente como investigador requiere de la evaluación y documentación de su desempeño, lo que hace que sea más consciente de su recorrido profesional, facilitándole aumentar su capacidad reflexiva y de autorregulación (Masip, Teixidor y Vilalta, 2011). La práctica reflexiva favorece un entorno de aprendizaje continuo, adaptado a las necesidades de los actuales sistemas educativos, que permite encontrar sentido a la evaluación como autorregulación y mejorar la capacidad profesional y la propia competencia como docentes (Gairín, 2011).

Un profesional competente requiere de capacidad para trabajar en equipo que permita integrarle en el desarrollo de proyectos colectivos, refuerzo de la autonomía y de la responsabilidad personal, apertura a nuevas metodologías más efectivas, aprovechamiento de las oportunidades que plantean las Tecnologías de la Información y de la Comunicación (TIC), una disposición permanente a la reflexión y a la investigación sobre la propia práctica docente, y la adaptación a una creciente demanda social orientada hacia una mayor profesionalización (Consejo Escolar del Estado, 2015). 


\section{a. El eportfolio como herramienta de evaluación auténtica}

La necesidad de formar al profesorado en competencias (Imbernón, 2007; Perrenoud, 2004; Tribó, 2008) supone seleccionar las estrategias y herramientas más adecuadas, pertinentes y congruentes que permitan una evaluación auténtica (Pérez Rendón, 2014). La herramienta más adecuada será aquella que propicia "la práctica continuada de la reflexión y la reflexión sobre la práctica" (Menéndez, 2013, p. 94), clave para la construcción del profesionalismo docente que mira por la calidad educativa y favorece ambientes de colaboración (Marcelo, 2001). La formación continua requiere de la documentación de la práctica docente haciéndole más consciente de su ejercicio a través de la reflexión y autorregulación, fomentado su autonomía y colaboración con otros. La recopilación y selección de experiencias de aprendizaje se deben trabajar con herramientas que evidencien dicho proceso, reflexionando sobre el mismo a la vez que visibilizan el desarrollo de habilidades y destrezas. Para Mateo y Martínez (2008) el portfolio es el procedimiento más popular dentro de la evaluación alternativa porque: a) es eficaz para la gestión de aprendizajes, b) permite analizar el grado de aprendizaje de los estudiantes a través de la recogida de evidencias, y c) valora los trabajos de manera global e integrada. Del uso de las TIC en la elaboración del portfolio, surge el concepto de portfolio digital o eportfolio (en adelante), definido como "un instrumento de colección digital que incluye demostraciones, recursos y logros individuales, de grupo, de la comunidad, organización o institución" (Lorenzo e Ittelson, 2005, p. 2) y está dirigido a la planificación del desarrollo personal y profesional (Zubizarreta, 2009). Fernández-Fernández, Arias-Blanco, Fernández-Alonso, Burguera-Condon y Fernández-Raigoso (2016) indican que el profesional reflexivo debe adaptarse a un modelo investigador y activo, además de estar organizado en red en torno a proyectos formativos vinculados al centro o a la materia docente.

La estructura básica para la realización de un eportfolio tendría cuatro fases: recopilación de evidencias, selección, reflexión y publicación (figura 1).

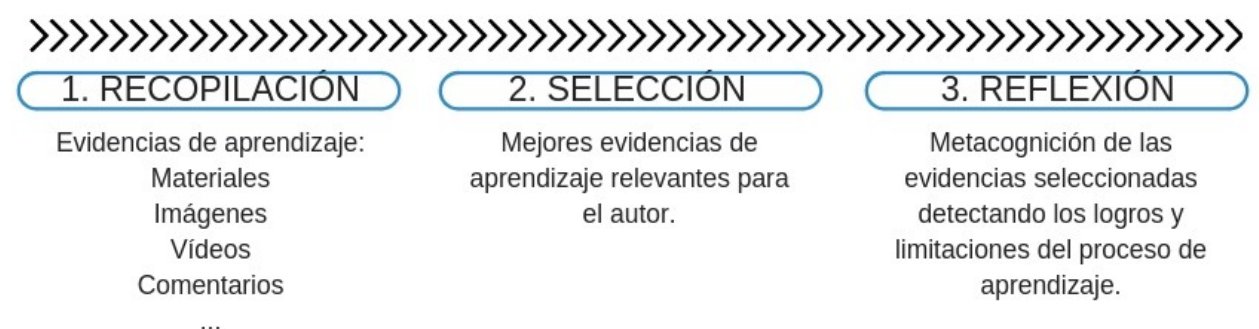

\section{PUBLICACIÓN DEL EPORTFOLIO}

Organización de las evidencias y reflexiones en un producto final.

Figura. 1. Fases de elaboración de la herramienta eportfolio Fuente: Elaboración propia

La primera fase, parte de una recopilación previa de evidencias que demuestran el aprendizaje, entendiendo que cuanto más relevante sea la evidencia más útil será valorarlo (Klenowski, 2014). Para evitar caer en una mera recopilación de evidencias, es necesaria la segunda fase, la selección. La selección crítica de las evidencias de entre todas las recopiladas en la fase anterior demuestran el logro de estándares específicos (Barrett, 2000). En la fase de selección "se trata de demostrar aquello que se sabe hacer y de la mejor manera" (Barberá, 2006, p. 500) para ello se tienen que elegir entre las evidencias más relevantes para su aprendizaje. Este proceso lleva asociado una 
práctica reflexiva, que permite detectar aquellas dificultades, problemas o debilidades, así como las ventajas surgidas de la evidencia seleccionada (Barberá, 2006). Sin esta reflexión continua no se puede considerar un proceso completo de elaboración (Sobrados, 2016) y es esto, precisamente, lo que le diferencia de otra herramienta utilizada en la evaluación del desarrollo profesional, los diarios. Cabero, López y Llorente (2012) subrayan que en la fase de selección es necesaria la reflexión y construcción de conocimientos, ayuda en la planificación de las tareas educativas, proporciona responsabilidad e introspección individual, y permite el seguimiento del proceso de aprendizaje del estudiante gracias a los comentarios vertidos en el mismo y una evaluación formativa.

Por último, hay que organizar las evidencias y elaborar el producto final (Barrett, 2000), es decir, la presentación final del producto, la cual se va realizando de forma paralela al resto de fases, dando seguimiento y continuidad a su elaboración. En este sentido, la última fase culminaría con la presentación del eportfolio como producto final que permitiría una recopilación de evidencias y reflexiones hacia una mejora de la práctica docente.

El eportfolio se adapta a la formación docente caracterizada por las "oportunidades para desarrollar y analizar el pensamiento y la actitud de los docentes en situaciones basadas en la experiencia y orientadas hacia los problemas y que incluyen o simulan actos reales" (Darling-Hammond y Snyder, 2000, p. 524). La vinculación entre la teoría y la práctica hacen del eportfolio una herramienta de evaluación auténtica y adecuada para la reflexión (Martínez Lirola, 2008). Esta idea del eportfolio como evaluación auténtica es apoyada por otros autores (Arraiz, Sabirón, Berbegal y Falcón, 2013; Barrett, 2009; Díaz Barriga y Pérez Rendón, 2010) que ven como la herramienta facilita "el proceso de enseñanza-aprendizaje recopilando muestras de las actividades de aprendizaje en momentos clave y realizando una reflexión sobre los logros y dificultades para la consecución de las competencias genéricas y específicas propuestas" (Gallego, Cacheiro, Martín y Angel, 2009, p. 2). Este tipo de evaluación se concibe como una actividad para la identificación de necesidades del desarrollo profesional en búsqueda de estrategias docentes que den solución a las mismas (Gómez Gonzalvo, 2006). Esta herramienta puede facilitar distintas formas de reflexión al analizar su enseñanza, desde la teoría y la práctica de manera conjunta (Toom, Husu y Patrikainen, 2015).

\section{b. El eportfolio y el desarrollo de competencias genéricas}

Esta herramienta se vincula al aprendizaje constructivista (Strudler y Wetzel, 2005) principalmente porque favorece la reflexión, la comunicación y la colaboración (Barberá, Gewerc y RodríguezIllera, 2009) aportando mayor protagonismo a la persona en formación. Se utiliza con mayor frecuencia en el ámbito universitario (Alcaráz, 2016; Barberá, Gewerc y Rodríguez-Illera, 2009; Rubio y Galván, 2013) favoreciendo un entorno activo y colaborativo, donde la práctica reflexiva destaca como su característica principal.

El eportfolio se utiliza para el aprendizaje y evaluación de competencias (Rodríguez-Donaire y Amante, 2010) generales a todas las titulaciones, destacando el desarrollo de competencias como la capacidad de reflexión, análisis crítico y un mayor control del trabajo realizado por cada persona (Barragán, 2005) o en grupo. Pérez Burriel (2010) utiliza el eportfolio del estudiante como instrumento para la evaluación de la actividad reflexiva. Parece que el eportfolio contribuye a mejorar significativamente en habilidades relacionadas con la autorregulación metacognitiva, el pensamiento crítico, la elaboración, el ensayo, la orientación intrínseca de los objetivos, la 
regulación del esfuerzo, la autoeficacia para el aprendizaje y el rendimiento y el valor de la tarea (Nguyen y Ikeda, 2015). Romero (2008) señala que esta herramienta permite la adquisición de competencias de aprender a aprender porque aumenta la autonomía del estudiante en su aprendizaje, le hace asumir responsabilidades, desarrolla la capacidad de autorregularse, y la toma de decisiones sobre sus aprendizajes.

En cuanto a la competencia de trabajo en equipo, Gámiz-Sánchez, Gallego-Arrufat y Crisol-Moya (2016) proponen la creación de un eportfolio de aprendizaje colaborativo. El desarrollo de competencias como la autonomía, el pensamiento crítico y la reflexión son también destacadas por autores que utilizan el portfolio de forma colaborativa (Estellés-Miguel, Barberá y Dema, 2013; Jordana y Sánchez, 2010; Romero, 2008; Wang, 2009). Los estudiantes valoran positivamente el uso del eportfolio en el trabajo en equipo (Nieto-Escámez, Ruiz-Muñoz y Moreno-Montoya, 2010) y tienen predisposición a colaborar en el futuro (Wang, 2009). El uso de la evaluación por pares permite valorar el eportfolio de otros compañeros, aunque se desconoce si se fomenta la conexión y mejora la calidad de la comunicación a largo plazo (Bolliger y Shepherd, 2010).

En definitiva, el uso del eportfolio del estudiante tiene una amplia trayectoria en Educación Superior, dando soporte al modelo de aprendizaje por competencias. En este sentido parece que el eportfolio contribuye al desarrollo de competencias genéricas a cualquier titulación, como son la competencia reflexiva, razonamiento crítico, trabajo en equipo y autonomía. Toom, Husu y Patrikainen (2015) concluyeron que el uso del eportfolio en formación inicial docente permitía posicionarse a los estudiantes en un nivel más general como futuros maestros y establecer objetivos a largo plazo para su desarrollo profesional. Se requiere de alternativas para el uso de la herramienta acorde al aprendizaje a lo largo de la vida profesional y adquirir las competencias necesarias (Guasch, Guàrdia y Barberà, 2009) para el profesorado.

La mejora educativa podría estar vinculada al desarrollo profesional docente; el eportfolio podría proporcionar al docente la reflexión necesaria para su ejercicio profesional, la autoevaluación que le permita guiar y mejorar continuamente sus procedimientos educativos y el aprendizaje de sus estudiantes. Esto plantea la necesidad de extender la herramienta a la educación obligatoria, priorizando la etapa de desarrollo profesional por ser un proceso de aprendizaje activo a lo largo de toda la vida profesional, desde la formación inicial hasta la autoformación del profesorado (Tejada Fernández, 2013) que apoye a los docentes en el desarrollo de competencias durante toda su trayectoria profesional. Hoekstra y Crocker (2015), subrayan la necesidad de más estudios para determinar qué elementos deben integrar el eportfolio para que apoye el desarrollo profesional y la evaluación del ejercicio docente. Por su parte, Guàrdia, Maina, Barberà y Alsina (2015) señalan que integrar esta herramienta en el proceso de formación va más allá del ámbito estrictamente formal y académico, así mismo hace más consciente al docente de cómo se aprende, por qué y cómo pueden aplicar los conocimientos en sus aulas, además de facilitar un mayor seguimiento del aprendizaje.

\section{Metodología}

La presente investigación con la herramienta eportfolio se ha desarrollado en un ámbito de formación para centros educativos concertados repartidos en 15 Comunidades Autónomas inmersos en procesos de cambio e innovación educativa, en concreto el programa "Profesores en acción". Este programa lleva asociado la realización de un curso online, donde los participantes 
construyen el eportfolio en base a los contenidos de los módulos presenciales guiados por el feedback de tutores expertos en la herramienta que les orientan y animan, bien resolviendo sus dudas en los foros correspondientes o bien, a través de la puntuación y comentarios que obtienen del seguimiento del eportfolio.

El objetivo principal de esta investigación es valorar si el programa de formación propuesto ha resultado eficaz y ha provocado cambios en la autopercepción de las competencias reflexiva, autonomía, razonamiento crítico y trabajo en equipo vinculadas al desarrollo profesional. Además, se abordan los siguientes objetivos específicos:

- Analizar la percepción del profesorado sobre la utilidad del eportfolio para el desarrollo profesional antes y después de su elaboración y valorar las posibles limitaciones.

- Comprobar si el eportfolio mejora la autopercepción de las competencias reflexiva, autonomía, razonamiento crítico y trabajo en equipo a través de la comparación antes y después de la formación de eportfolio.

- Plantear propuestas de mejora para el desarrollo profesional del docente mediante el uso del eportfolio.

La muestra tiene un carácter incidental, dado que se ha construido conforme a la matriculación de los participantes en el programa de formación. Está integrada por un total de 306 personas (profesorado de educación infantil, primaria, secundaria y bachillerato), de los cuales 151 pertenecen a la primera promoción (curso 2014-2015) y 155 a la segunda promoción (curso 20152016). El tipo de diseño es cuasi-experimental con dos tratamientos, pretest y postest (tabla 1).

\begin{tabular}{|l|c|c|c|c|}
\hline \multicolumn{1}{|c|}{ Promoción } & Pretest & Tratamiento1 & Tratamiento2 & Postest \\
\hline Primera & $\mathrm{O}$ & $\mathrm{X}$ & & $\mathrm{O}$ \\
\hline Segunda & $\mathrm{O}$ & & $\mathrm{X}$ & $\mathrm{O}$ \\
\hline
\end{tabular}

Tabla 1. Diseño cuasi-experimental

Fuente: Elaboración propia

En la primera promoción, el curso online se desarrolla en una plataforma virtual privada y estática que impide su personalización. En cambio, en la segunda promoción la plataforma online donde se aloja el curso tiene un diseño más cercano al profesorado y utiliza la herramienta gratuita Moodle. En ambas plataformas se recogen los contenidos y videotutoriales del curso de eportfolio junto con actividades correspondientes a cada módulo del curso presencial sobre innovación educativa. Cada actividad dispone de una fecha límite de entrega, antes de la asistencia al siguiente módulo presencial. La elaboración del eportfolio es de carácter obligatorio en la primera promoción, mientras que en la segunda es voluntaria, tratando de eliminar la presión generada de la experiencia en el primer tratamiento.

En la primera promoción, los docentes tienen la posibilidad de seleccionar entre dos herramientas para elaboran el eportfolio. A través de la herramienta digital gratuita Google Sites que permite crear un espacio web adaptada a docentes con un nivel medio-bajo de competencia digital, como el caso de la muestra seleccionada, o la herramienta Mahara considerada "un puente entre el uso abierto de los recursos digitales y la administración institucionalizada de los portfolios de los estudiantes a través del sistema de gestión del aprendizaje" (Castaño, 2014, p.100). Google Sites permite la personalización del eportfolio y presenta pocas diferencias con otras herramientas como Mahara más dirigidas a la elaboración de esta herramienta (San José, 2017). En este sentido, en la 
segunda promoción se pasa a incluir Google Sites como una única herramienta de realización del eportfolio, aunque los participantes tienen posibilidad de seleccionar la que más les interese.

Se miden las variables competencia reflexiva, razonamiento crítico, trabajo en equipo y autonomía ligadas al desarrollo profesional y necesarias para afrontar la práctica diaria. La eficacia docente viene determinada por la autopercepción de las competencias genéricas objeto de estudio y la utilidad del eportfolio mide la autopercepción de los docentes sobre la utilidad, las ventajas y limitaciones de su elaboración.

Se opta por un modelo mixto como procedimiento de recogida de los datos (Onwuegbuzie y Leech, 2006) donde se combinan en la misma etapa, tanto métodos cuantitativos y cualitativos para evitar ser excesivamente reduccionista (Hmelo-Silver, 2003). Estos métodos agregan cierta complejidad al diseño del estudio pero integrando todas las ventajas de cada uno de los enfoques (Hernández, Fernández y Baptista, 2003). Se realiza una estrategia secuencial explicativa (Creswell, 2008, citado en Pereira, 2011) donde el orden queda definido por los resultados cuantitativos, utilizando los resultados cualitativos para explicar e interpretar los datos.

La competencia digital necesaria, para la elaboración del eportfolio y para el manejo de la plataforma del curso online, se mide a través de un cuestionario online construido ad hoc. Para su desarrollo, se toma como referencia el Marco Común de Competencia Digital Docente (MCCDD) elaborado por el INTEF (2013), seleccionando aquellas áreas competenciales más acordes a la elaboración y manejo de contenidos digitales.

Se diseña un segundo cuestionario "Competencias genéricas y utilidad del eportfolio" con el propósito de valorar la definición previa de un portfolio, la autopercepción de las competencias de estudio y su utilidad. El pretest permite tener un conocimiento en profundidad de las competencias y habilidades necesarias para seguir el curso online. Este fue sometido a una evaluación interjueces con una consistencia interna aceptable con ítems con una puntuación (Theta de Armor) por encima de 0.70 (Armor, 1973). Una vez validado el cuestionario y recogidos los primeros datos de docentes se procede a la reelaboración del cuestionario sobre competencias genéricas para la recogida de datos en el postest, donde se añaden además las posibles dificultades que hayan podido tener en la elaboración de la herramienta.

Los análisis cuantitativos incluyen pruebas no paramétricas, en concreto prueba de los signos para dos muestras relacionadas, para comparar la autopercepción de competencias genéricas y utilidad del eportfolio entre el prestest y el postest realizadas con el programa SPSS v. 25. Los análisis cualitativos se utilizan para el análisis de las respuestas a las preguntas abiertas de los cuestionarios y los foros de entrega de actividades y consultas de las dos plataformas correspondientes a cada promoción del curso online de eportfolio, mediante el programa MAXQDA 12.

El formato de recogida de datos es digital porque el uso de estas herramientas "han revolucionado las antiguas formas de recogida de datos hasta el punto de que están quedando obsoletas" (Lorca, Carrera y Casanovas, 2016, p. 92). Las ventajas que ofrecen se refieren al escaso conocimiento técnico y tiempo para su elaboración, bajo coste económico, respuestas en tiempo real, inserción de contenido multimedia, almacenaje de respuestas automáticas y eliminación de posibles errores en la introducción manual de respuestas (Alarco y Álvarez-Andrade, 2012). 


\section{Resultados}

Se presentan los resultados obtenidos tras el uso de la herramienta eportfolio en la formación docente teniendo en cuenta: la percepción del profesorado sobre la utilidad de la herramienta, las ventajas y limitaciones encontradas y la autopercepción de la mejora de las competencias genéricas asociadas al desarrollo profesional docente. Todo ello lleva a una propuesta final del uso de la herramienta.

\section{a. Percepción del profesorado sobre la utilidad de la herramienta de eportfolio}

Tras la realización de los análisis cualitativos facilitados por las respuestas abiertas del cuestionario "Competencias genéricas y utilidad del eportfolio" al finalizar la formación, los docentes manifiestan que el eportfolio es de utilidad porque puede ser una herramienta de apoyo en el aula para la evaluación de los estudiantes y la práctica docente a través del conocimiento de otras prácticas. Además, el uso del eportfolio podría ser útil para el desarrollo profesional porque consideran que mejora la capacidad reflexiva y crítica, aportando sentido a las futuras acciones docentes. A continuación, se indican algunas valoraciones de los docentes sobre el uso del eportfolio en el espacio de observaciones del cuestionario. Los profesores valoran las posibilidades de la herramienta: "Es verdad que, viendo los portfolios de algunos compañeros en la coevaluación, se me ha quedado mucho por definir e incluso contar con más evidencias de cada módulo" (Profesor 5). Y la visibilidad del trabajo docente: "Me ha parecido muy útil e interesante a pesar de no haberlo podido finalizar por falta de tiempo y motivos personales, la posibilidad de ver ejemplos prácticos ha sido muy enriquecedora. Útil para recopilar evidencias, ponerlas en común y facilitar aprendizajes colaborativos" (Profesor 6 ).

Los docentes indican que la herramienta les ayuda en su práctica porque puede ser de utilidad con los estudiantes: "Creo que la creación de portfolios con los alumnos de primaria y secundaria puede ser muy atractiva para ellos" (Profesor 2); porque aumenta la relación con los estudiantes: "Me parece una herramienta muy potente para nuestra labor docente y para la relación con nuestros alumnos." (Profesor 3); fomentar la autonomía de su trabajo diario: "La actividad me parece muy interesante, tanto como herramienta individual como para llevarla a cabo con mis alumnos" (Profesor 1); tomando conciencia de cómo aprenden y como eportfolio sumativo: "También me ha resultado muy positivo el apartado dedicado a la autoevaluación y coevaluación como método que experimentar con los chicos/as", es decir como producto final de una asignatura, como indica este participante: "El portfolio educativo me parece una herramienta excelente para que los alumnos puedan evidenciar sus esfuerzos, logros y progresos en un tema o materia, así como reflexionar sobre su progreso durante el proceso de enseñanza / aprendizaje. Me parece una alternativa muy útil de evaluación del trabajo final del alumno." (Profesor 4).

\section{b. Ventajas y limitaciones en la elaboración del eportfolio}

El elemento principal que ha limitado la elaboración del eportfolio ha ido la falta de tiempo personal y profesional, es decir aquel del que disponen durante y después de su jornada laboral. Esto lleva a pensar que el tiempo del que disponen tanto fuera como dentro del aula es escaso. Hay que tener en cuenta que casi la totalidad de la muestra nunca había tenido experiencia previa en el uso de esta herramienta, la falta de tiempo y estrés asociado podría pensar que el tiempo de dedicación sea mayor por el desconocimiento de la herramienta como se manifiesta en este comentario: 
"Agobio, porque no conocía el portfolio, y tampoco disponía de mucho tiempo para hacerlo" (Profesor 7), aunque siguen valorando la herramienta como positiva: "No lo realicé por falta de tiempo para dedicarle, pero no porque lo vea como una herramienta inútil, al contrario creo que es una herramienta muy positiva" (Profesor 8).

En el caso de la segunda promoción, además de la falta de tiempo personal el análisis cualitativo también señala la baja competencia digital, la recopilación de evidencias y la falta de reflexión. En cuanto a la limitación de la competencia digital, ambas promociones parten de una competencia digital media-baja, teniendo mayores dificultades en compartir información y en el desarrollo de contenidos. En general, parece haber un nivel de competencia digital insuficiente en docentes de educación obligatoria, también observada por Pérez Escoda y Rodríguez Conde (2016), más concretamente en la dificultad de compartir recursos a través de herramientas digitales y edición y elaboración de recursos. Es necesario, como afirman Keenan, Rosenberg, Greenhalg y Koehler (2016) más investigaciones para explorar el potencial de los eportfolios docentes para fomentar la aplicación de la tecnología a su práctica docente y mejorar su competencia digital.

\section{c. Autopercepción de las competencias genéricas tras la elaboración del eportfolio}

El análisis de los resultados permite concluir que los docentes perciben que la herramienta eportfolio es útil en su desarrollo profesional porque facilita el conocimiento de otras experiencias educativas y la capacidad de autocrítica. Perciben que su uso facilita el desarrollo de algunos aspectos de la competencia reflexiva y de razonamiento crítico.

Para cada una de las competencias se han analizado las diferencias entre el pretest y el postest teniendo en cuenta los ítems incluidos en cada una. Se ha aplicado la prueba de los signos para dos muestras relacionadas. Diferenciamos ambas promociones dado que como describimos se han producido cambios en la intervención.

En las tablas 2 y 3 se observan diferencias entre el pretest y postest de la competencia reflexiva, indicando los ítems correspondientes.

\begin{tabular}{|c|c|c|c|c|c|c|c|}
\hline & $\begin{array}{l}\text { post1_a - } \\
\text { pre1_a }\end{array}$ & $\begin{array}{l}\text { post1_b - } \\
\text { pre1_b }\end{array}$ & $\begin{array}{l}\text { post1_c }- \\
\text { pre1_c }\end{array}$ & $\begin{array}{l}\text { post1_d - } \\
\text { pre1_d }\end{array}$ & $\begin{array}{l}\text { post1_e - } \\
\text { pre1_e }\end{array}$ & $\begin{array}{ll}\text { post1_f }_{\text {pre1_f }} & -\end{array}$ & $\begin{array}{l}\text { post1_g - } \\
\text { pre1_g }\end{array}$ \\
\hline $\bar{Z}$ & -.658 & -2.228 & -2.500 & -1.460 & .000 & -.567 & -1.701 \\
\hline $\begin{array}{l}\text { Sig. asintót. } \\
\text { (bilateral) }\end{array}$ & .511 & .026 & .012 & .144 & 1.000 & .571 & .089 \\
\hline \multicolumn{8}{|c|}{$\begin{array}{l}\text { a) Necesito un tiempo para abordar la solución a un problema educativo que se me plantee. } \\
\text { b) Al terminar la jornada escolar, vuelvo a pensar sobre ella y reflexiono sobre lo que ha ocurrido. } \\
\text { c) Anoto aquello que quiero mejorar sobre mi práctica diaria (docente o directiva), para valorar los cambios } \\
\text { después de un tiempo. } \\
\text { d) Valoro distintas alternativas para planificar la concreción del currículo oficial. } \\
\text { e) Una vez tomada una decisión, valoro la eficacia de la misma en mi práctica docente o directiva. } \\
\text { f) Puedo dar argumentos sobre las decisiones que tomo. } \\
\text { g) Antes de tomar una decisión, reflexiono y valoro las diferentes opciones. }\end{array}$} \\
\hline
\end{tabular}

Tabla 2. Estadísticos de contraste de la competencia reflexiva en la primera promoción.

Fuente: Elaboración propia

Los valores de la prueba de los signos de la tabla $2(p-v<.05)$ muestra cambios significativos en los ítems de la competencia reflexiva que permiten reflexionar después de la práctica docente y anotar qué se quiere mejorar sobre la práctica diaria. 


\begin{tabular}{|l|l|l|l|l|l|l|l|}
\hline & $\begin{array}{l}\text { post2_a- } \\
\text { pre2_a }\end{array}$ & $\begin{array}{l}\text { post2_b- } \\
\text { pre2_b }\end{array}$ & $\begin{array}{l}\text { post2_c- } \\
\text { pre2_c }\end{array}$ & $\begin{array}{l}\text { post2_d- } \\
\text { pre2_d }\end{array}$ & $\begin{array}{l}\text { post2_e- } \\
\text { pre2_e }\end{array}$ & $\begin{array}{l}\text { post2_f- } \\
\text { pre2_f }\end{array}$ & $\begin{array}{l}\text { post2_g- } \\
\text { pre2_g }\end{array}$ \\
\hline Z & -4.857 & -.857 & -3.203 & -2.811 & -.676 & & -1.373 \\
\hline $\begin{array}{l}\text { Sig. } \\
\text { asintót. } \\
\text { (bilateral) }\end{array}$ & $\mathbf{. 0 0 0}$ & .391 & $\mathbf{. 0 0 1}$ & $\mathbf{. 0 0 5}$ & .499 & & .170 \\
\hline $\begin{array}{l}\text { Sig. exacta } \\
\text { (bilateral) }\end{array}$ & & & & & $.405^{*}$ & \\
\hline \multicolumn{2}{|l}{ "Se ha usado la distribución binomial. } & & & & & \\
\hline
\end{tabular}

Tabla 3. Estadísticos de contraste de la competencia reflexiva en la segunda promoción Fuente: Elaboración propia

En la segunda promoción (tabla 3), se observan cambios significativos en los ítems relacionados con el tiempo necesario para abordar una solución y la valoración de distintas alternativas, aunque en este caso no han sido positivos debido probablemente a que se percibieron con altos niveles de competencia al inicio de la formación (pretest).

En las tablas 4 y 5 se observan las diferencias entre el pretest y postest de la competencia razonamiento crítico.

\begin{tabular}{|c|c|c|c|c|c|c|c|}
\hline & $\begin{array}{l}\text { post1_s- } \\
\text { pre1_s }\end{array}$ & $\begin{array}{l}\text { post1_t- } \\
\text { pre1_t }\end{array}$ & $\begin{array}{l}\text { post1_u- } \\
\text { pre1_u }\end{array}$ & $\begin{array}{l}\text { post1_v- } \\
\text { pre1_v }\end{array}$ & $\begin{array}{l}\text { post1_w- } \\
\text { pre1_w }\end{array}$ & $\begin{array}{l}\text { post1_x- } \\
\text { pre1_x }\end{array}$ & $\begin{array}{l}\text { post1_y-y- } \\
\text { pre1_y }\end{array}$ \\
\hline Z & -2.229 & -.189 & -1.784 & -1.765 & -.718 & -1.741 & -.183 \\
\hline $\begin{array}{l}\text { Sig. asintót. } \\
\text { (bilateral) }\end{array}$ & .026 & .850 & .074 & .078 & .472 & .082 & .855 \\
\hline $\begin{array}{l}\text { s) Contrasto } \\
\text { t) Analizo sit } \\
\text { u) Utilizo la a } \\
\text { v) Soy crítico } \\
\text { w) Valoro los } \\
\text { x) Evalúo los } \\
\text { y) Valoro lo }\end{array}$ & $\begin{array}{l}\text { dez y crec } \\
\text { ies y elijo } \\
\text { aluación c } \\
\text { igo mism } \\
\text { y contras } \\
\text { sos educa } \\
\text { aprendid }\end{array}$ & $\begin{array}{l}\text { dad de la } \\
\text { lejor decis } \\
\text { hte mi tra } \\
\text { rante mi } \\
\text { Ina situac } \\
\text { s antes de } \\
\text { Irante el } p\end{array}$ & $\begin{array}{l}\text { entes cons } \\
\text { al objetivo } \\
\text { diario. } \\
\text { ajo. } \\
\text { para tomar } \\
\text { lizarlos en } \\
\text { eso para a }\end{array}$ & $\begin{array}{l}\text { la decisión } \\
\text { ción de un } \\
\text { arlo a una }\end{array}$ & $\begin{array}{l}\text { ecuada. } \\
\text { criterios pr } \\
\text { ación simi }\end{array}$ & terminado & \\
\hline
\end{tabular}

Tabla 4. Estadísticos de contraste de la competencia razonamiento crítico en la primera promoción Fuente: Elaboración propia

La tabla 4 muestra valores significativos, en la primera promoción, tras la elaboración del eportfolio en el ítem relacionado con contrastar la validez y credibilidad de las fuentes.

\begin{tabular}{|l|l|l|l|l|l|l|l|}
\hline & $\begin{array}{l}\text { post2_s- } \\
\text { pre2_s }\end{array}$ & $\begin{array}{l}\text { post2_t- } \\
\text { pre2_t }\end{array}$ & $\begin{array}{l}\text { post2_u- } \\
\text { pre2_u }\end{array}$ & $\begin{array}{l}\text { post2_v- } \\
\text { pre2_v }\end{array}$ & $\begin{array}{l}\text { post2_w- } \\
\text { pre2_w }\end{array}$ & $\begin{array}{l}\text { post2_x- } \\
\text { pre2_x }\end{array}$ & $\begin{array}{l}\text { post2_y- } \\
\text { pre2_y }\end{array}$ \\
\hline Z & -1.857 & & -1.500 & -.183 & -2.373 & -.530 & -.385 \\
\hline $\begin{array}{l}\text { Sig. asintót. } \\
\text { (bilateral) }\end{array}$ & .063 & & .134 & .855 & .018 & .596 & .700 \\
\hline $\begin{array}{l}\text { Sig. exacta } \\
\text { (bilateral) }\end{array}$ & & $1.000^{*}$ & & & & & \\
\hline
\end{tabular}

Tabla 5. Estadísticos de contraste de la competencia razonamiento crítico en la segunda promoción Fuente: Elaboración propia 
En la segunda promoción (tabla 5) los docentes perciben que tras elaborar el eportfolio valoran los pros y contras de una situación para tomar una decisión adecuada.

Sin embargo, los análisis realizados no muestran diferencias significativas en la percepción del profesorado de educación obligatoria sobre la mejora de las competencias de autonomía y trabajo en equipo con la elaboración del eportfolio.

\section{d. Propuesta de intervención en formación docente}

Los resultados ponen de manifiesto que el eportfolio puede ser una herramienta útil a los docentes de educación obligatoria en ejercicio porque es necesario prácticas activas que contribuyan a la unión de teoría y práctica diaria y, sobre todo, les ayude a la reflexión de ambos aspectos valorando alternativas de trabajo dirigidas a mejorar. Parece conveniente pues plantear una propuesta formativa para el uso de la herramienta eportfolio en educación obligatoria. Como dice Lewis (2017), el propósito del eportfolio se debe fijar al inicio de la formación junto con los objetivos del uso de la herramienta y los criterios de evaluación e incluir la herramienta como parte del diseño del aprendizaje para los resultados definidos.

En base a las ventajas y limitaciones encontradas se plantea que el propósito del eportfolio se debe fijar al inicio de la formación junto con los objetivos del uso de la herramienta y los criterios de evaluación. La elaboración del eportfolio requiere del diseño de unas actividades de aprendizaje constructivistas que incluyan el sentido propio del eportfolio y guiar su aprendizaje hacia la mejora de la práctica docente. Para eso, las actividades de elaboración de la herramienta deben tener en cuenta el tiempo del que dispone el profesorado y estar diseñadas de tal manera que puedan adaptarlo a su ritmo y orientarlo a una adecuada práctica reflexiva. Para evitar la excesiva carga docente con eportfolio individuales, se propone la posibilidad de explorar el diseño de los eportfolios de aprendizaje grupal, como proponen Gámiz-Sánchez, Gallego-Arrufat y Crisol-Moya (2016). En este caso, los mismos docentes del equipo presentarían las mismas características profesionales, en cuanto a nivel y departamento al que pertenecen. Por otro lado, para evitar que el eportfolio se convierta en una simple herramienta de recopilación de materiales, es importante, como señalan Díaz Barriga y Pérez Rendón (2010), que dispongan de instrumentos que permitan la autoevaluación y la reflexión. Se propone el uso de rúbricas siguiendo otras experiencias (Tur y Urbina, 2014) y hojas de reflexión compartidas con los compañeros con criterios consensuados.

Se debe tener en cuenta el nivel de competencia digital y debido a que elabora en formato digital debemos promover cursos de formación con TIC que sitúen el conocimiento de los docentes en el centro del proceso (Bairral y Santos, 2012). Además, se requiere de una mayor orientación y retroalimentación adecuada del profesor-tutor para guiarles, tal y como se ha demostrado en otras experiencias (Heinrich, Bhattacharya y Rayudu, 2007). No obstante, se precisa de un mayor entrenamiento para la tutorización y "disponer al menos de dos evaluadores independientes y entrenados, que procedan de acuerdo a unos criterios acordados y niveles de desempeño que resulten claros y precisos y así permitan analizar discrepancias y lograr consensos" (Díaz Barriga y Pérez Rendón, 2010, p. 22). Por último, sería necesario facilitar sesiones de apoyo y retroalimentación que guíen al profesorado a llevar a la práctica las reflexiones que ha ido proponiendo en el eportfolio (Tucker, Stronge, Gareis y Beers, 2003). 


\section{Conclusiones}

El desarrollo profesional docente precisa de herramientas que permitan el aprendizaje en competencias básicas para su labor educativa, sean curriculares o pedagógicas, como las 11 identificadas en el proyecto ProficiencyIn+E (2015). Pero es la reflexión y la investigación sobre la práctica las que permiten detectar dificultades y buscar soluciones. El eportfolio se distingue por proporcionar un medio de documentar, seleccionar y reflexionar sobre el propio proceso de enseñanza-aprendizaje, muy introducido en educación superior y poco en educación obligatoria. Si este trabajo es relevante es porque evalúa el eportfolio en la formación de docentes en ejercicio de etapas obligatorias, prueba una herramienta que la teoría asimila a la evaluación auténtica y favorece un proceso continuo y actualizado de reflexión crítica que podría relacionarse positivamente con el desarrollo profesional (Marcelo y Vaillant, 2009). El uso del eportfolio resulta significativo en el desarrollo de las competencias reflexiva y razonamiento crítico, de forma similar a Barrett (2000) y Rubio y Galván (2013), además de fomentar el aprendizaje activo en el proceso de enseñanza aprendizaje, junto con una actitud de autocrítica relacionando teoría y práctica, como señala Bozu (2012). Sin embargo no se observan cambios en las competencias de autonomía y trabajo en equipo. Posiblemente porque el nivel inicial de partida en estas competencias era ya alto antes del programa formativo, similar a lo que indican Rivilla, Garrido y Domínguez (2010) y porque no se han diseñado estrategias de colaboración específicas.

El programa de formación $y$, particularmente, el uso del eportfolio, teniendo en cuenta la percepción del profesorado, es útil y eficaz para mejorar competencias clave del desarrollo profesional porque facilita el conocimiento de otras experiencias educativas y la capacidad de autocrítica; también encuentran algunas dificultades para su elaboración como es la falta de tiempo, igual que Barberá (2005), Corominas (2000) y Vázquez-Cano, López Meneses y Jaén Martínez (2017) y la baja competencia digital. No obstante, es necesario realizar un seguimiento, por un lado, para comprobar si el continuo uso de la herramienta mejoraría las competencias genéricas implicadas en el desarrollo profesional y por otro lado, si el uso del eportfolio permite un mejor traslado del contenido aprendido en el programa de formación y su eficacia en la mejora del rendimiento y aprendizaje de los estudiantes. Murillo y Krichesky (2012) proponen al menos dos cursos escolares para que se produzca una institucionalización de la innovación en el centro, el primer año iría dirigido a la implementación de la innovación tras el aprendizaje recibido y el segundo a producir mejoras.

Estos resultados plantean líneas de mejora que apoyan la idea de utilizar el eportfolio como una herramienta de aprendizaje en un contexto de formación docente donde se lleven a cabo prácticas activas que contribuyan a la unión de la teoría y su práctica diaria que orienten la reflexión. Requiere de una orientación y retroalimentación adecuada del profesor-tutor para guiarles en todo el proceso de aprendizaje, imprescindible para el apoyo y seguimiento continuo. Por ello, se debe considerar el apoyo de un asesor pedagógico, experto en innovación, tecnología y portfolio que facilite el procedimiento a los docentes novatos. La coevaluación ha servido para visualizar el trabajo de otros compañeros, aprender otras experiencias y ser conscientes de cómo estaban elaborando la herramienta, pero ha sido insuficiente para el desarrollo de la competencia de trabajo en equipo, posiblemente porque no se han elaborado eportfolios colaborativos. Se abre una nueva línea sobre el eportfolio de aprendizaje colaborativo, que permita utilizar la herramienta en equipo y eficazmente el trabajo individual, la reflexión y la toma de decisiones conjunta. 
Futuros estudios deberían ir dirigidos a crear herramientas donde los docentes puedan desarrollar competencias adecuadas para llevar a cabo la innovación educativa, entre ella la autonomía y el trabajo en equipo, así como evaluar el impacto del uso del eportfolio docente en el aprendizaje del estudiantado.

\section{Agradecimientos}

Este trabajo ha sido realizado en el marco del proyecto financiado por el Ministerio de Economía y Competitividad, Secretaría de Estado de Investigación, Desarrollo e Innovación, Dirección General de Investigación Científica y Técnica (PROFICIENCyIn+E. Evaluación de Competencias Docentes para la inclusión y la excelencia. EDU2015-63844-R).

\section{Referencias}

Alarco, J. J. y Álvarez-Andrade, E. V. (2012). Google Docs: una alternativa de encuestas online. Educación Médica, 15(1), 9-10. doi:10.4321/s1575-18132012000100004

Alcaráz, N. (2016). La Evaluación a través de Portafolios: ¿Una Ocasión para el Aprendizaje? Revista Iberoamericana de Evaluación Educativa, 9(1), 31-46. doi:10.15366/riee2016.9.1.002

Armor, D. J. (1973). Theta reliability and factor scaling. Sociological methodology, 5, 17-50.

Arraiz, A., Sabirón, F., Berbegal, A. y Falcón, C. (2013). La evaluación de competencias: el portafolio digital. La Cuestión Universitaria, 8, 140-151.

Bairral, A.M. y Santos, R.T. (2012). E-Portfolio improving learning in mathematics pre-service. Digital Education Review, 21, 1-12.

Barberá, E. (2005). La evaluación de competencias complejas: la práctica del portafolio. Revista Educere, $9(31), 497-504$.

Barberá, E. (2006). Aportaciones de la tecnología a la e-Evaluación. RED. Revista de Educación a Distancia, 5(6), 1-13. doi:10.6018/red/50/4

Barberá, E., Gewerc, A. y Rodríguez-Illera, J. L. (2009). Portafolios electrónicos y educación superior en España: Situación y tendencias [Monográfico]. RED, Revista de Educación a Distancia, 3, 1-13. doi: https://doi.org/10.6018/red/50/7

Barragán, R. (2005). El Portafolio, metodología de evaluación y aprendizaje de cara al nuevo Espacio Europeo de Educación Superior. Una experiencia práctica en la Universidad de Sevilla. Revista Latinoamericana de Tecnología Educativa, 4(1), 121-139.

Barrett, H. (2000). Electronic Teaching Portfolios: Multimedia Skills + Portfolio Development = Powerful Professional Development. Association for the Advancement of Computing in Education (AACE), 1-7. Recuperado de http://electronicportfolios.com/portfolios/site2000.html

Barrett, H. (2009). Balancing the Two Faces of ePortfolios. Recuperado de http://electronicportfolios.org/balance/

Bozu, Z. (2012). Cómo elaborar un portafolio para mejorar la docencia universitaria: una experiencia de formación del profesorado novel. Barcelona: Octaedro/ ICE-UB.

Bolliger, D. U. y Shepherd, C. E. (2010). Student perceptions of ePortfolio integration in online courses. Distance Education, 31(3), 295-314. doi: 10.1080/01587919.2010.513955

Cabero, J., López, E. y Llorente, M. C. (2012). E-Portafolio universitario como instrumento didáctico 2.0 para la reflexión, evaluación e investigación de la práctica educativa en el espacio europeo de educación superior. Virtualidad, Educación y Ciencia, 3(4), 27-46.

Cabero, J., Llorente, E. y Morales-Lozano, J.A. (2018). Evaluación del desempeño docente en la formación virtual: ideas para la configuración de un modelo. RIED. Revista Iberoamericana de Educación a Distancia, 21(1), 261-279. doi:10.5944/ried.21.1.17206

Castaño, A. X. (2014). The Application of Eportfolio in Higher Education: implications for Student Learning. UniversitasTarraconensis. Revista de Ciències de l'Educació, 1(2), 99-103. doi: $10.17345 /$ ute.2014.2.462 
Carbonell, J. (2012). La aventura de innovar: el cambio en la escuela. Madrid: Morata.

Consejo Escolar del Estado (2015). El profesorado del siglo XXI. XXI Encuentro de Consejos Escolares Autonómicos y del Estado. Madrid: Ministerio de Educación, Cultura y Deporte. Recuperado de http://ntic.educacion.es/cee/jpa/21encuentro/index.html.

Corominas, E. (2000). ¿Entramos en la era portafolio? Bordón. Revista de pedagogía, 52(4), 509-522.

Day, C. (1999). Developing Teachers: The Challenges of Lifelong Learning.London: Psychology Press.

Darling-Hammond, L. y Snyder, J. (2000). Authentic Assessment of Teaching in Context. Teaching and Teacher Education, 16(5), 523-545.

Díaz Barriga, F. y Pérez Rendón, M. M. (2010). El portafolio docente a escrutinio: sus posibilidades y restricciones en la formación y evaluación del profesorado. Observar. Revista electrónica de Observatorio sobre la Didáctica de las Artes, 4, 6-27.

Estellés-Miguel, S. M., Barberá, T. y Dema, C. M. (2013). Aplicación del portafolio grupal en la asignatura de Dirección de Producción y Logística: resultados de una prueba piloto. Educade: revista de educación en contabilidad, finanzas y administración de empresas, 4, 124-138.

Fernández-Fernández, S., Arias-Blanco, J.M., Fernández-Alonso, R., Burguera-Condon, J. y Fernández-Raigoso, M. (2016). Pensamiento reflexivo e investigador en Educación. Aspectos a tener en cuenta en la formación del profesorado. RELIEVE, Revista Electrónica de Investigación y Evaluación Educativa, 22(2). doi:10.7203/relieve.22.2.8425

Gallego, D., Cacheiro, M. L., Martín, A. M. y Angel, W. (2009). El ePortfolio como estrategia de enseñanza y aprendizaje. Edutec. Revista Electrónica de Tecnología Educativa, 30, 1-12. doi:10.21556/edutec.2009.30.444

Gairín, J. (2011). Formación de profesores basada en competencias. Bordón. Revista de Pedagogía, 63(1), 93108.

Gámiz-Sánchez, V.M., Gallego-Arrufat, M.J. y Crisol-Moya, E. (2016). Impact of electronic portfolios on prospective teachers' participation, motivation and autonomous learning. Journal of Information Technology Education: Research, 15, 517-533. doi:10.28945/3575

Gómez Gonzálvo, C. (2006). Evaluación del desarrollo profesional docente: una necesidad del territorio. Revista Electrónica de las Ciencias Médicas en Cienfuegos, 4(2), 43-46.

González Maura, V. G. (2006). El diario como instrumento de diagnóstico y estimulación del desarrollo profesional del profesorado. Revista iberoamericana de educación, 38(2), 1-15.

Guasch, T., Guàrdia, L. y Barberà, E. (2009). Prácticas del portafolio electrónico en el ámbito universitario del Estado Español. Revista de Educación a Distancia-RED [Monográfico VIII], 1-11.

Guàrdia, L., Maina, M., Barberà, E. y Alsina, I. (2015). Matriz conceptual sobre usos y propósitos de los eportfolios. Procedia, Social and Behavioral Sciences, 196, 106-112. doi: 10.1016/j.sbspro.2015.07.019

Heinrich, E., Bhattacharya, M. y Rayudu, R. (2007). Preparation for lifelong learning using ePortfolios.European Journal of Engineering Education, 32(6), 653-663. doi: 10.1080/03043790701520602

Hernández, R., Fernández, C. y Baptista, P. (2003). Metodología de la investigación (3a ed.). México: Editorial Mc Graw-Hill.

Hmelo-Silver, C. E. (2003). Analyzing collaborative knowledge construction: multiple methods for integrated understanding. Computers \& Education, 41(4), 397-420. doi:10.1016/j.compedu.2003.07.001

Hoekstra, A. y Crocker, J.R. (2015). Design, implementation, and evaluation of an ePortfolio approach to support faculty development in vocational education. Studies in Educational Evaluation, 46, 61-73. doi:10.1016/j.stueduc.2015.03.007

Iglesias. M.J., Lozano, I. y Roldán, I. (2018). La calidad e innovación educativa en la formación continua docente: un estudio cualitativo en dos centros educativos. Revista Iberoamericana de Educación, $77(1), 13-34$.

Imbernón, F. (1999). El desarrollo profesional del profesorado de Primaria. Revista internacional de Investigación e Innovación Educativa, 1(2), 59-68.

Imbernón, F. (2007). 10 Ideas Clave. La formación permanente del profesorado: Nuevas ideas para formar en la innovación y el cambio (Vol. 4). Barcelona: Graó.

Jordana, J. y Sánchez, F. J. (2010). Cooperative work and continuous assessment in an Electronic Systems laboratory course in a Telecommunication Engineering degree. En IEEE EDUCON 2010 Conference (pp. 395-400). doi: 10.1109/EDUCON.2010.5492551 
Keenan, S. F., Rosenberg, J., Greenhalg, S. y Koehler, M. J. (2016). Examining Teachers' Technology Use Through Digital Portfolios. Actas de la Society for Information Technology \& Teacher Education International Conference. Association for the Advancement of Computing in Education (AACE) (pp. 1084-1091). Savannah, GA, United States, March 21-26.

Klenowski, V. (2014). Desarrollo del portafolios para el aprendizaje y la evaluación: procesos y principios. Madrid: Narcea.

Lewis, L. (2017). ePortfolio as pedagogy: Threshold concepts for curriculum design. E-Learning and Digital Media, 14(1-2), 72-85.

Lorenzo, G. e Ittelson, J. (2005). An overview of e-portfolios. Educause Learning initiative, 1, 1-27.

Lorca, S., Carrera, X. y Casanovas, M. (2016). Análisis de herramientas gratuitas para el diseño de cuestionarios on-line. Píxel-Bit, Revista de Medios y Educación, (49), 91-104. doi:10.12795/pixelbit.2016.i49.06

Marcelo, C. (2001). Aprender a enseñar para la sociedad del conocimiento. Revista complutense de educación, 12(2), 531-593.

Martínez Lirola, M. (2008). Una propuesta de evaluación en el EEES: el uso del portfolio en una clase de idiomas. Porta Linguarum, 9, 23-34.

Masip, M., Teixidor, M. y Vilalta, D. (2011). Acompañar procesos de cambio a través de la formación. Cuadernos de pedagogía, 417, 74-76.

Mateo, J. y Martínez, F. (2008). La evaluación alternativa de los aprendizajes. Cuadernos de docencia universitaria. Barcelona: Ediciones Octaedro.

Marcelo, C. y Vaillant, D. (2009). Desarrollo profesional docente: ¿Cómo se aprende a enseñar? Madrid: Narcea.

Menéndez, J. L. (2013). La carpeta docente como instrumento de evaluación del profesorado universitario. ¿Es posible valorar la mejora de la calidad de la enseñanza? En I. Arbesú y F. Díaz Barriga (Coords.), Portafolio docente. Fundamentos, modelos y experiencias (pp. 87-110). México: UAM-X, Ediciones Díaz de Santos.

Murillo, F. J. y Krichesky, G. J. (2012). El proceso del cambio escolar. Una guía para impulsar y sostener la mejora de las escuelas. REICE. Revista Iberoamericana sobre Calidad, Eficacia y Cambio en Educación, $10(1), 26-43$.

Nieto-Escámez, F. A., Ruiz-Muñoz, A. M. y Moreno-Montoya, M. (2010). Un ejemplo de portafolio electrónico para el trabajo en grupo basado en la plataforma WebCT-Blackboard. RISTI: Revista Ibérica de Sistemas e Tecnologias de Informação, 5, 17-30.

Nguyen, L. T. y Ikeda, M. (2015). The effects of ePortfolio-based learning model on student self-regulated learning. Active Learning in HigherEducation, 16(3), 197-209.

Onwuegbuzie, A. J. y Leech, N. L. (2006). Linking Research Questions to Mixed Methods Data Analysis Procedures. Qual Report, 11(3), 474-498.

Perrenoud, P. (2004). Diez nuevas competencias para enseñar. Barcelona: Graó.

Pérez Burriel, M. (2010). La evaluación de la actividad reflexiva a partir del portafolio del estudiante. REP:TE. Revista de Enseñanza de la Psicología: Teoría y Experiencia, 6(1), 1-12.

Pérez Escoda, A. y Rodríguez Conde, M. J. (2016). Evaluación de las competencias digitales autopercibidas del profesorado de educación primaria en Castilla y León. Revista de Investigación Educativa, 34(2), 399415. doi:10.6018/rie.34.2.215121

Pérez Rendón, M. M. (2014). Evaluación de competencias mediante portafolios. Perspectiva Educacional, 53(1), 19-35.

Pereira, Z. (2011). Los diseños de método mixto en la investigación en educación: Una experiencia concreta. Revista Electrónica Educare, 1, 15-29.

PROFICIENCyIn+E (2015). Evaluación de Competencias Docentes para la inclusión y la excelencia. Proyecto MINECO, EDU2015-63844-R, 2016-2018.

Rivilla, A. M., Garrido, M. C. D. y Domínguez, M. M. (2010). Evaluación de las competencias docentes. Innovación educativa, 10(53), 19-41.

Rodríguez-Donaire, S. y Amante, B. (2010). Portfolio digital: un nuevo método de evaluación de competencias. Universidad Europea. Recuperado de http://abacus.universidadeuropea.es/handle/11268/1862 
Rodríguez-Illera, J. L., Galván, C. y Martínez, F. (2013). El portafolio digital como herramienta para el desarrollo de competencias transversales en el alumnado. Education in the Knowledge Society (EKS), 14(2), 157-177.

Romero, C. (2008). El portafolio y el trabajo de grupo: una experiencia del crédito ECTS en la formación del magisterio especialista en Educación Física. Revista Interuniversitaria del Formación del Profesorado REIFOP, 11(2), 73-84.

Rubio, M. J. y Galván, C. (2013). Portafolios digitales para el desarrollo de competencias transversales. Aportaciones principales de los estudios con Carpeta Digital en el marco del grupo de investigación Ensenyament i Aprenentatge Virtual. Digital Education Review, 24, 53-68.

San José, D. L. (2017). Evaluating, Comparing, and Best Practice in Electronic Portfolio System Use. Journal of Educational Technology Systems, 45(4), 476-498. doi: 10.1177/0047239516672049

Sobrados, M. (2016). El trabajo docente en grupos numerosos. Experiencias en el uso del portafolio. Opción, 32(10), 773-788.

Strudler, N. y Wetzel, K. (2005). The diffusion of electronic portfolios in teacher education: Issues of initiation and implementation. Journal of Research on Technology in Education, 37(4), 411-433. doi:10.1080/15391523.2005.10782446

Tejada Fernández, J. (2013). Profesionalización docente en la universidad: implicaciones desde la formación. RUSC. Universities and Knowledge Society Journal, 10(1), 170-184.

Tribó, G. (2008). El nuevo perfil profesional de los profesores de secundaria. Educación XX1, 11, 183-209. doi:10.5944/educxx1.11.0.314

Toom, A., Husu, J. y Patrikainen, S. (2015). Student teachers' patterns of reflection in the context of teaching practice. European Journal of Teacher Education, 38(3), 320-340. doi: $10.1080 / 02619768.2014 .943731$

Tucker, P. D., Stronge, J. H., Gareis, C. R. y Beers, C. S. (2003). The efficacy of portfolios for teacher evaluation and professional development: Do they make a difference? Educational administration quarterly, 39(5), 572-602. doi:10.1177/0013161x03257304

Tur, G. y Urbina, S. (2014). Blogs as Eportfolio Platforms in Teacher Education: Affordances and Limitations Derived from Student Teachers' Perceptions and Performance on their Eportfolios. Digital Education Review, 26, 1-23.

Vázquez-Cano, E., López Meneses, E. y Jaén Martínez, A. (2017). The Group e-portfolio to improve TeachingLearning Process at University.Journal of e-Learning and Knowledge Society, 13(2), 65-76.

Villardón, M. L. (2006). Evaluación del aprendizaje para promover el desarrollo de competencias. Educatio siglo $X X I, 24,57-76$.

Wang, C. X. (2009). Comprehensive Assessment of Student Collaboration in Electronic Portfolio Construction: An Evaluation Research. TechTrends, 53(1), 58-66. doi:10.1007/s11528-009-0238-1

Zubizarreta, J. (2009). The learning portfolio. San Francisco: Anker. 